\title{
Hipercorrección grafémica en importaciones léxicas. Datos del español bonaerense
}

\author{
Yolanda Hipperdinger - Universidad Nacional del Sur - CONICET (Argentina) \\ yhipperdinger@uns.edu.ar
}

Rebut / Received: 2-11-15

Acceptat / Accepted: 15-3-16

Resum. Hipercorrecció grafèmica en importacions lèxiques. Dades de l'espanyol de Buenos Aires. En aquest article analitzem la hipercorrecció verificada en l'escriptura d'importacions lèxiques provinents de llengües europees de caràcter internacional a partir d'escrits obtinguts de la base de dades que recull textos de l'àrea dialectal de Buenos Aires de l'espanyol de l'Argentina. Observem que aquesta hipercorrecció assumeix gairebé exclusivament la forma d'una amplificació grafèmica que, al seu torn, consisteix generalment en una duplicació consonàntica. Explorem les raons d'aquesta preferència i concloem que es deu al fet que l'amplificació registrada no enfosqueix la relació amb les respectives realitzacions orals més esteses. També hem observat que en tots els casos els recursos emprats en l' "exageració" referida formen efectivament part de les convencions de les llengües donants, de manera que no impliquen una altra innovació que la posicional.

Paraules clau: importacions lèxiques, representació escrita, hipercorrecció, espanyol de Buenos Aires.

Abstract. Graphemic hypercorrection in lexical imports. Data from Buenos Aires Spanish. We analyze in this article hypercorrection found in the writing of lexical imports from European languages of international level. The analysis is made on the basis of writings obtained from the database that includes texts from the dialectal area of Buenos Aires Spanish. We observe that such hypercorrection almost exclusively takes the form of a graphemic amplification that, in turn, generally consists of a consonant duplication. We explore the reasons for this preference and conclude that the registered amplification is preferred because it does not darken the relationship with the respective most widespread oral realizations. We have also observed that in all cases 
the resources used in the referred "exaggeration" are effectively part of the conventions of donor languages, so they do not involve another innovation but the positional one.

Keywords: lexical imports, written representation, hypercorrection, Buenos Aires Spanish.

\section{Introducción}

Un lugar central en la atención de los estudiosos del contacto lingüístico se reserva para el trasvase de unidades léxicas, que constituye el emergente más común de la coexistencia interlingüística, sea directa o indirecta (Winford, 2003, p. 26), y suele referirse como transferencia "en significado y forma” (Gómez Capuz, 1997, p. 84 — traducción nuestra_; Gómez Molina, 2000, p. 329). En el marco del estudio de las importaciones léxicas ${ }^{1}$, a su vez, uno de los aspectos más atendidos es el de su adaptación a las pautas de la lengua receptora, tanto a nivel gramatical como a nivel fónico y —en compleja relación con ambos tipos de adaptación — también a nivel grafémico.

Sobre la base de datos del área dialectal bonaerense del español de la Argentina ${ }^{2}$, en este artículo nos ocuparemos de las configuraciones grafémicas de las importaciones léxicas, pero no para analizar su adaptación: nos interesa, por el contrario, explorar la falta de adaptación que recurrentemente se registra en ellas, incluso cuando su empleo se encuentra socialmente extendido. En particular, nos ocupará la manifestación más evidente del intento de conservación: la hipercorrección en la escritura de las voces importadas, i.e. la exageración de la extranjeridad que resulta de la combinación de la voluntad mimética que los mismos usos transparentan con un conocimiento insuficiente de las configuraciones que se pretende reproducir ${ }^{3}$.

En indagaciones concretas sobre el uso escrito de importaciones léxicas, los mecanismos de la adaptación se enfocan sistemáticamente ${ }^{4}$; la desadaptación que la hipercorrección

1. Empleamos con valor hiperonímico la designación importación léxica, que recoge el aporte basal de Haugen (1950, p. 214), porque permite tanto distinguir claramente el ingreso en "forma y significado" de otros emergentes del contacto vinculados (en especial el del calco) como reservar el rótulo de préstamo para ingresos léxicos de amplia difusión en la lengua receptora, de acuerdo con su acepción más común (Haspelmath, 2009, pp. 41-42).

2. Por la clasificación dialectal implicada, véase Fontanella de Weinberg (2000). Sobre el español bonaerense, su caracterización y evolución, véase especialmente Fontanella de Weinberg (1987).

3. La caracterización que ofrecemos toma como antecedente la empleada hace ya décadas, en relación con la adaptación fonológica, por Weinreich ([1953] 1968, pp. 27-28).

4. Constituyen ejemplo de ello los trabajos — en relación, en todos los casos, con la escritura de anglicismos en diversas variedades de español— de Reyes Padilla (2009), Giménez Folqués (2011) y Mateescu (2013). 
supone, en cambio, aun cuando es ocasionalmente consignada ${ }^{5}$, no es objeto de atención per se. Como lo ha señalado Winter-Froemel, los que llama hiper-extranjerismos (hyperforeignisms) "are not mentioned in most traditional and current studies of loanwords" (Winter-Froemel, 2008, p. 166). No obstante, revisten el mayor interés: cada forma hipercorrecta constatada es evidencia del intento precautorio que ha guiado a quien la produjo (que quiso precaverse de adaptar), así como de las "ideas acerca de lo que es "correcto"' (Iturrioz, 2006, p. 15) que han impulsado su intento. Desde esa perspectiva - distante de la prescriptiva desde la cual a veces la hipercorrección y otros fenómenos semejantes se denuncian-, intencionamos con este trabajo contribuir al desarrollo de una atención específica a la hipercorrección en la representación escrita de las importaciones léxicas.

\section{La adaptación grafémica como constancia de asentamiento}

La (completa) adaptación grafémica ha sido considerada el síntoma por antonomasia de la integración (igualmente completa) de una incorporación en significado y forma a la lengua receptora ${ }^{6}$. Así, por ejemplo, Castillo Carballo (2011, p. 64) afirma:

Las voces de procedencia foránea se incorporan a nuestra vida diaria de una forma casi imperceptible. Desde que empezamos a oírlas por primera vez, las adoptamos y se insertan en nuestros discursos tanto orales como escritos. Aunque la verdadera constancia de que estos vocablos están asentados en nuestra lengua es cuando se configura su fisonomía gráfica.

La razón de que la adaptación grafémica pueda erigirse en constancia de asentamiento radica en la diferencia general entre los usos orales y los escritos de las importaciones léxicas, por un lado, y en el carácter del contacto, por otro.

En relación con la primera diferencia anotada, las pautas de la lengua receptora constituyen el eje gravitacional de la adaptación que se constata corrientemente en la oralidad: la fonología recipiente y los hábitos articulatorios respectivos ejercen una presión tal que, incluso cuando se intenta una aproximación a la pronunciación de origen, el intento normalmente resulta filtrado por esas pautas, como lo muestran innumerables estudios, desde la contribución pionera de Weinreich ([1953] 1968) hasta

5. Véanse por ejemplo las observaciones de Corbella Díaz y Real Cairós (1997, p. 235) y de Rodríguez González (2008, pp. 279-280).

6. Entendemos aquí por integración "el grado en que una palabra es sentida como miembro pleno del sistema de la lengua recipiente" (Haspelmath, 2009, pp. 42-43; traducción nuestra), asentado en la extensión de su uso. 
producciones actuales como la de Cabré Monné (2010). La escritura, en cambio, no está sujeta a esa presión: de hecho, para dar cuenta de la escasa atención comparativa dispensada tradicionalmente a los procesos de adaptación grafémica se ha aducido su mayor dependencia relativa de "factores no estructurales" (Gómez Capuz, 1998, p. 233).

En cuanto al carácter del contacto, cuando las importaciones ingresan de manera directa, a través de la coexistencia efectiva de las lenguas implicadas en situaciones de bilingüismo social, los hablantes nativos o dominantes de la lengua receptora tienen como modelo disponible para la respectiva realización oral (exista o no un modelo correlativo para la realización escrita) el que les proveen los hablantes nativos o dominantes de la lengua donante; en cambio, cuando las importaciones se difunden por contacto indirecto, a través del transporte de novedades y productos sin interacción oral entre los portadores y los receptores, la vía escrita por la que ingresan solo deja en disponibilidad como referencia las correspondientes configuraciones de origen.

El resultado de la coincidencia de la mayor libertad de la escritura con la disponibilidad del modelo de la lengua de origen hace que, en los ingresos léxicos por contacto indirecto, pueda registrarse en la oralidad una adaptación ausente en la escritura. Así, por ejemplo, De Baere (2012, p. 47) afirma que "la adaptación fonológica no implica automáticamente una adaptación en el ámbito ortográfico”, y Leal Cabrera (2001, p. 2) concluye que en el español de Cuba anglicismos como thank you, volleyball, shopping o shoot han tendido en la oralidad a "acomodarse a la realización más cercana del español" pero que, simultáneamente, "mantienen su grafía original".

Cuando el productor particular intenta mantener la configuración grafémica de origen puede obviamente conseguirlo, si sabe cómo. Puede también, sin embargo, generar grafías mixtas o parcialmente adaptadas, por transferencia de pautas grafémicas de la lengua con cuya escritura está más familiarizado. Si procura asegurarse de no adaptar, puede asimismo producir formas hipercorrectas, que resultan en consecuencia incuestionables indicadores de los imperativos actitudinales subyacentes y merecen, por lo mismo, atención detenida.

\section{Alternantes hipercorrectos en español bonaerense}

Como lo anticipamos, constatamos la producción de formas hipercorrectas en la escritura de vocablos de procedencia foránea en la variedad territorial bonaerense del español. Para la recolección de los datos que expondremos nos limitamos al área léxica de la gastronomía, especialmente permeable a la neologización por la vía de la importación (Romaine, 1995, p. 142; Myers-Scotton, 2006, p. 213).

Constituimos nuestro corpus a través del reciente relevamiento de las ofertas de productos publicitadas in situ en setenta locales comerciales del rubro gastronómico de 
la ciudad de Bahía Blanca ${ }^{7}$. Registramos 119 piezas léxicas importadas con al menos una configuración grafémica no adaptada de modo completo, y para 29 de ellas (todas con la configuración de origen como modelo) relevamos alternantes que transparentan la operación de una hipercorrección. Esas formas hipercorrectas son las siguientes:

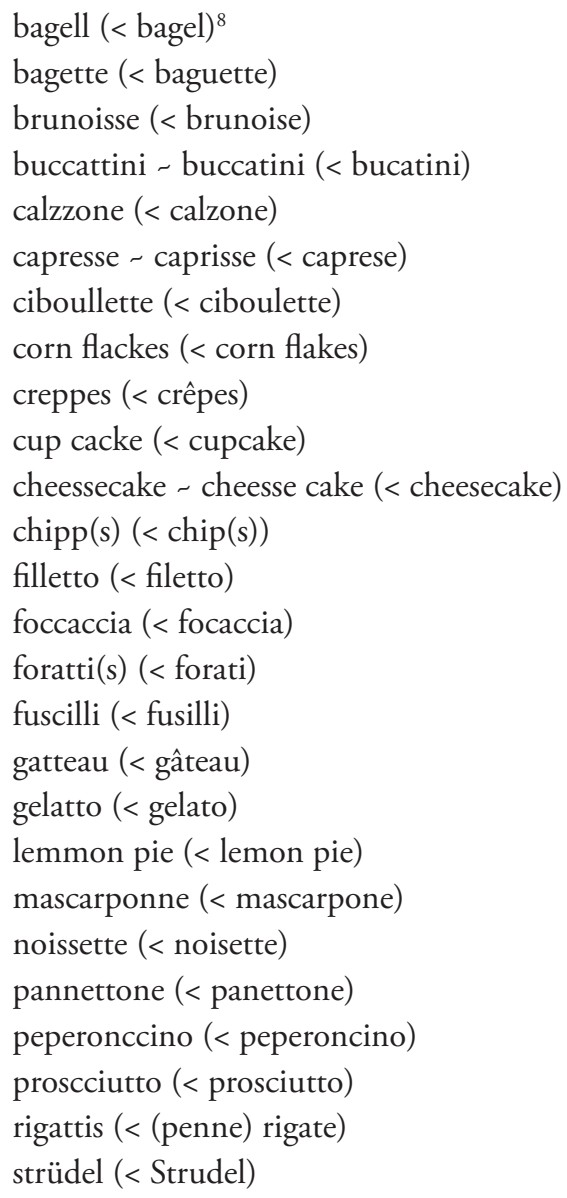

7. Bahía Blanca se ubica a unos 700 kilómetros al sur de la Ciudad Autónoma de Buenos Aires, capital de la Argentina, "y no resulta posible a primera vista distinguir lingüísticamente a los hablantes de una y otra ciudad” (Fontanella de Weinberg, Blanco de Margo, Hipperdinger, Rigatuso, Suardíaz de Antollini y Virkel de Sandler, 1991, p. 36). Los locales comerciales referidos se ubican en el centro y macrocentro bahienses, y se orientan todos a sectores medios.

8. Consignamos como configuración de origen, para esta especialidad de la cocina judía, la transliteración más difundida tanto en español como en otras lenguas, como el alemán y el inglés. En el caso que nos ocupa, esta última lengua puede tomarse como donante, ya que el consumo de la especialidad de panificación referida se ha extendido últimamente junto con el de otras de procedencia norteamericana. 
tagliattelle $(<$ tagliatelle)

tiramizzu $(<$ tiramisù $)$

vermiccelli $(<$ vermicelli)

Además de las formas enlistadas, registramos los alternantes boconccino, ciboullete, expresso, lazagna, noicette, roquefordy vermicceli, cuya clasificación como hipercorrecciones es dudosa:

a. En las representaciones grafémicas boconccino, ciboullete y vermicceli se constata el uso de un dígrafo, lo cual coincide - aunque no se trate del mismo-con las respectivas configuraciones de origen (bocconcino, ciboulette y vermicelli), por lo que puede tratarse de una simple metátesis.

b. En los casos de lazagna y noicette, el empleo de $z$ y $c$ en lugar de la $s$ de origen permite pensar que la selección obedece a un intento de corregir lo que el productor entiende como una adaptación al español, ya que $z$ y $c$ son menos frecuentes que $s$ para [s] en la variedad lingüística receptora, si bien existe igualmente la posibilidad de que la misma intercambiabilidad de los grafemas limite estos usos a errores ortográficos.

c. En el caso de expresso se han fundido el italiano espresso y el español expreso, por lo que no resulta claro que se trate de una hipercorrección de la configuración grafémica italiana.

d. En el caso de roqueford, por último, siendo el apellido y la marca de automotores Ford muy conocidos entre los hablantes de español bonaerense, puede pensarse que sobre su base se ha corregido analógicamente, quizá por etimología popular, la denominación de origen correspondiente.

En cuanto a las piezas léxicas implicadas, es de señalar que presentan diverso grado de extensión de uso, desde las que solo ocurren raramente, como gelato, hasta las de empleo corriente, como baguette $e^{9}$. Provienen, además, de diversas lenguas: del italiano (mayoritariamente), del francés, del inglés y del alemán.

\section{Qué se (hiper)corrige}

Los alternantes hipercorrectos constatados corresponden, como lo apuntamos, a importaciones léxicas de diversa difusión y de diverso origen, pero por encima de sus diferencias, todos corresponden a importaciones de lenguas europeas de carácter internacional y, por lo mismo, prestigiosas. Resulta fácil inferir que la desadaptación exige ese prestigio como conditio sine qua non: es la "preocupación por respetar la norma"

\footnotetext{
9. Aunque no nos ocuparemos de ello aquí, es de señalar que los alternantes hipercorrectos mismos pueden también ordenarse por su frecuencia: frente al carácter idiosincrásico que regularmente ostentan, algunos muestran cierta extensión de uso y uno en particular, capresse, se registra en el corpus aún más reiteradamente que la correspondiente configuración de origen.
} 
de la otra lengua la que conduce a "una restitución exagerada de las formas prestigiosas" (Álvarez González, 2006, p. 115).

Según se observa en el registro presentado, la hipercorrección grafémica puede implicar la operación de distintas estrategias:

1. la sustitución del grafema que el productor particular supone adaptado por el que presume que corresponde en la configuración de origen (como en el caso de la sustitución de $u$ por $\ddot{u}$ registrada en strüdel),

2. la reducción del número de grafemas si entiende que se ha filtrado una pauta española (como en la supresión de $u$ para grafiar $[\gamma]$ que se aprecia en bagette), o

3. la amplificación del número de grafemas si supone que se ha operado una simplificación (como en la duplicación de $c$ relevada en peperonccino).

No obstante, se observa igualmente la abrumadora preferencia de una de esas estrategias por sobre las restantes, ya que en la amplia mayoría de los casos la hipercorrección se verifica a través de una amplificación grafémica: $c c$ por $c$ en buccattini - buccatini, foccaccia, peperonccino, proscciutto y vermiccelli, ck por $k$ en corn flackes y cup cacke, ll por $l$ en bagell, ciboullette y filletto, $m m$ por $m$ en lemmon pie, $n n$ por $n$ en mascarponne y pannettone, $p p$ por $p$ en creppes y chipp(s), sc por $s$ en fuscilli, ss por $s$ en brunoisse, capresse - caprisse, cheessecake - cheesse cake y noissette, tt por $t$ en buccattini, foratti(s), gatteau, gelatto, rigattis y tagliattelle, y $z z$ por $z$ en calzzone $^{10}$. Como puede verse, además, esa estrategia preferida se aplica especialmente a grafemas consonánticos, por lo común procediendo a su duplicación.

Sobre la base de lo expuesto, la pregunta que se impone es por qué quienes exageran la extrañeza grafémica de las voces importadas lo hacen regularmente del modo señalado. Procuraremos acercarnos a la respuesta a ese interrogante deslindando dos cuestiones de diferente índole: la primera atañe a la relación entre las configuraciones escritas y las orales, i.e. a las correspondencias grafémico-fonológicas, y la segunda a las ideas subyacentes (Iturrioz, 2006, p. 15).

\section{Del impulso y la dirección}

\subsection{Correspondencias grafémico-fonológicas}

En relación con las correspondencias grafémico-fonológicas, en el tratamiento de las importaciones léxicas intervienen tanto las de la lengua receptora como las de las lenguas donantes. Al respecto, es pertinente la reiterada observación (estrechamente ligada al carácter del contacto, al que nos referimos supra) de que las voces importadas tienden a adaptarse de manera dispar según su vía de ingreso: las ingresadas por vía escrita tienden a ser pronunciadas siguiendo la pauta de correlación grafémico-fonológica de la lengua

10. En tiramizzu también hay amplificación, pero combinada con sustitución (zz por $s)$. 
receptora, mientras que en las ingresadas por vía oral tiende a imitarse la realización oral de origen ${ }^{11}$. La imitación de la pronunciación de origen suele suponer la interacción de los hablantes de la lengua receptora con hablantes de la lengua donante, por cuanto son sus realizaciones las que se constituyen en modelo. Contemporáneamente, no obstante, las configuraciones orales imitativas de piezas léxicas importadas pueden extenderse sin esa interacción, si las emplean actores sociales (principalmente, los comunicadores de los medios masivos) cuya habla puede tomarse igualmente como referencia. Este es frecuentemente el caso cuando se implica la pronunciación de importaciones léxicas procedentes de lenguas de prestigio, aun cuando la imitación sea solo aproximada y se limite a "reglas básicas de correspondencia entre sonido y grafía" (Gómez Capuz, 2001, p. 17).

La exposición simultánea de los hablantes no modélicos a estas configuraciones orales imitativas y a las escritas de origen ${ }^{12}$ puede conducir a la memorización de ciertas correspondencias grafémico-fonológicas puntuales, miméticas respecto de las de la lengua donante y distintas de las de la variedad lingüística receptora, que resultan así autonomizadas de las importaciones léxicas conocidas y pasan a constituirse en recursos para el tratamiento de otras, i.e. para (intentar) leerlas como en la lengua de origen y no como se haría de acuerdo con las correspondencias españolas.

En espańol bonaerense se han constatado tales correspondencias miméticas autonomizadas para importaciones léxicas del inglés, la lengua donante de la mayoría de las recientes, así como para incorporaciones procedentes de otras lenguas europeas prestigiosas; por consignar solo unos pocos ejemplos ${ }^{13}$, en piezas léxicas importadas del inglés los dígrafos ee y oo se corresponden respectivamente con $[\mathrm{i}] \mathrm{y}[\mathrm{u}]$, en voces del francés ou se corresponde con $[\mathrm{u}]$, y en importaciones del alemán y del inglés $h$ se corresponde con $[\mathrm{x}]$ (Hipperdinger, 2015). Si bien las correspondencias pueden ser transversales a más de una lengua donante, su especificidad se revela cuando no hay coincidencia en las respectivas lenguas de origen: ou se corresponde con [u] para voces provenientes del francés, pero se corresponde con [aw] para importaciones del inglés (como lo ejemplifican las extendidas realizaciones orales de las piezas léxicas que han preservado su escritura original mousse y mouse, procedentes respectivamente del francés y del inglés y convencionalmente pronunciadas [mús] y [máws]). La funcionalidad de tales correspondencias es evidente: no se requiere dominio del francés para trasladar el

11. Véanse al respecto Pratt (1980, p. 156) y Gómez Capuz (2001, pp. 10ss.) para su aplicación a los anglicismos, y Fontanella de Weinberg (1994, p. 72) para su aplicación a italianismos en español bonaerense.

12. Entre las piezas léxicas importadas implicadas en esa exposición ocupan un lugar de especial importancia los nombres propios - y en particular los apellidos-, por cuya inclusión en los estudios sobre adaptación se ha abogado reiteradamente (véanse e.g. las apreciaciones de Bajo Pérez, 2008, p. 7, y Cabré Monné, 2010, p. 9).

13. Si bien nos referimos a correspondencias grafémico-fonológicas, para transparentar mejor los usos efectivos ofrecemos transcripciones fonéticas. 
prestigio asociado a esa lengua a los usos particulares de sus importaciones (y en último término al hablante), sino que alcanza con hacer esfuerzos mínimos como recordar que ou se lee $[\mathrm{u}]$.

La afirmación con la que iniciamos este apartado, así, puede reformularse diciendo que, en el tratamiento de que son objeto los vocablos de procedencia foránea por parte del hablante común, pueden intervenir tanto las correspondencias grafémico-fonológicas de la lengua receptora como las miméticas respecto de las lenguas donantes que hayan llegado a autonomizarse en el sentido aludido.

\subsection{Lectura española y correspondencias miméticas}

Como ya lo señalamos, la mayor parte de los alternantes hipercorrectos registrados corresponde a piezas léxicas procedentes del italiano. En el tratamiento fónico de importaciones léxicas de esa lengua, lo regular en español bonaerense es la simplificación de las consonantes dobles de origen (inexistentes en español), aun cuando en las formas más extendidas en la escritura se mantenga la duplicación consonántica (Lasry, 2014). De entre las correspondencias referidas, lo dicho vale incluso para la correspondencia $l l$-[1], en la que la simplificación se ha operado sobre la diferencia entre una posible lectura española y la italiana, a favor de esta última. Esa regularidad vuelve irrelevante la presencia de tales duplicaciones en las configuraciones escritas de origen en relación con la realización oral correspondiente más extendida en la variedad lingüística receptora. Por lo mismo, no tiene consecuencias sobre dicha relación la amplificación registrada en buccattini - buccatini y foccaccia (cc por $c$ ante vocal no anterior), filletto (ll por $l$ ), mascarponne y pannettone ( $n n$ por $n$ ), y buccattini, foratti(s), gelatto, rigattis y tagliattelle $(t t$ por $t)$.

Por otra parte, en la realización oral de voces provenientes del italiano se halla bastante extendida en espańol bonaerense una correspondencia puntual entre $c c$ ante vocal anterior y la consonante africada prepalatal sorda, correspondencia que recupera la de la lengua de origen; las ocurrencias de peperonccino y vermiccelli son una prueba de ello, como también lo son las de boconccino y vermicceli $i^{14}$.

En cambio, la correspondencia italiana entre sc seguida de vocal anterior y una consonante fricativa prepalatal no ha sido objeto de una convencionalización mimética puntual en español bonaerense: en la posición apuntada, sc sistemáticamente se lee [s]; un buen ejemplo lo constituye el apellido de quien fuera vicepresidente de la Argentina y gobernador de la provincia de Buenos Aires, Daniel Scioli, cuya pronunciación regular en la variedad lingüística de que nos ocupamos es [sjóli]. La aplicación de la pauta de

14. Esa correspondencia, sin embargo, no es estrictamente estable, i.e. su grado de convencionalización no es máximo (Lasry, 2014), con lo que puede vincularse la forma proscciutto que relevamos. 
lectura española en este caso subyace a la grafía hipercorrecta relevada fuscilli, que se correlaciona por lo tanto, al igual que la forma escrita de origen, con la pronunciación más extendida ([fusíli $]$ ).

Del mismo modo, no tiene tampoco correlato en español ni ha sido objeto de una convencionalización puntual mimética la oposición entre sibilantes dentales que se corresponde en italiano con la diferencia grafémica entre $s$ y ss intervocálicas. La correlación se opera con [s] en ambos casos en la realización oral de voces importadas del italiano en español bonaerense, y en esa indiferenciación se asienta la grafía hipercorrecta capresse.

Por último, y en vistas de que el español desconoce la africación dental, es de destacar asimismo que si bien en español bonaerense el dígrafo italiano $z z$ puede registrarse ocasionalmente en correspondencia con [ts], en particular en el caso de ciertas importaciones léxicas interlingüísticamente difundidas (pizza, mozzarella), no se trata de una correspondencia extendida; son muestra de ello las pronunciaciones de los apellidos de conocidas figuras públicas en los que, por el contrario, se correlaciona $z z$ con [s] de modo regular, como en el caso de Florencio Randazzo, ex Ministro del Interior y Transporte de la Nación, del influyente dirigente agropecuario Eduardo Buzzi o del exitoso futbolista Ezequiel Lavezzi. Realizado así por lo común el dígrafo zz según la pauta de lectura española, $z$ italiana puede duplicarse en $z z$ en calzzone, y hasta $s$ puede volverse $z z$ en tiramizzu.

De lo que llevamos dicho se infiere la general irrelevancia de la duplicación consonántica inscripta en las configuraciones grafémicas de origen para el tratamiento fónico de las piezas léxicas importadas en español bonaerense, ya que se la verifica aun en el tratamiento de voces provenientes de una lengua en la que la duplicación tiene sistemáticamente un correlato en la oralidad. Son excepciones las correspondencias miméticas señaladas entre $c c$ ante vocal anterior y la consonante africada prepalatal sorda y entre $l l$ y $[1]$, ambas convencionalizadas aunque en diverso grado; esta última correspondencia, especialmente estable, se aplica en español bonaerense a la realización oral de importaciones léxicas no solo del italiano sino también del alemán, del inglés y (de parte de las) del francés, y está en la base de la reposición constatada en nuestro registro tanto en bagell como en ciboullette (y ciboullete).

En cuanto a las amplificaciones grafémicas registradas para vocablos procedentes del inglés $(m m, p p$, ss y $c k)$ y del francés ( $p p$ y $s s)$, se procede respecto de ellas del mismo modo que respecto de las consonantes dobles italianas, por lo que esas amplificaciones tampoco tienen consecuencias sobre la relación entre las configuraciones grafémicas "intervenidas" y las configuraciones orales correspondientes.

En síntesis, lo expuesto evidencia que quienes hipercorrigen lo hacen de modo privilegiado a través de la amplificación grafémica porque, por las razones citadas, en el conjunto de los casos esta no altera la correspondencia con la realización oral más extendida para la importación léxica de que se trate. En cambio, la reducción grafémica 
verificada en bagette (< baguette) sí oscurece la relación con la configuración oral regular [bayé(t)], ya que por un lado no existe una correspondencia mimética autonomizada de $g$ ante vocal anterior con una consonante velar ${ }^{15} y$, por otro lado, el resultado de la lectura espańola tampoco se ajusta a la configuración oral referida. Algo semejante puede decirse de la sustitución grafémica operada en strüdel (< Strudel): si bien la incorporación de la diéresis no afecta la lectura española (que únicamente la reconoce para el caso de $g$ ante vocal anterior), contrasta con la extendida correspondencia mimética entre $\ddot{u}$ y el diptongo [ju], que refleja desagregadamente los rasgos de anterioridad y abocinamiento de la vocal alemana ${ }^{16}$.

\subsection{Los rasgos característicos como guía}

Ahora bien, la formulación que acabamos de presentar atiende a condiciones de posibilidad, pero abarca opciones cuya actualización no se registra en el uso. Al respecto, resulta patente que lo que ocurre como amplificación efectivamente forma parte de las pautas grafémicas de las respectivas lenguas donantes: para extranjerizar la escritura no se emplean cualesquiera rasgos ajenos a la propia lengua, sino solamente los que se reconocen como característicos de una lengua determinada. En tal sentido, y como puede verse en las ocurrencias enlistadas, la innovación implicada en la amplificación consonántica no va más allá de una reubicación ${ }^{17}$.

Los sitios en los que se opera la hipercorrección que analizamos, así, reflejan qué se reconoce como propio y distintivo de la otra grafía, lo cual se evidencia en el hecho de que cuando un recurso grafémico se percibe como característico de una lengua ocurre solo en piezas léxicas provenientes de ella (como $z z$ y $\ddot{u}$ en voces del italiano y del alemán, respectivamente).

En consecuencia, si el prestigio de las lenguas donantes y la confianza en su apropiación le otorgan al productor particular el impulso para intentar apegarse a las configuraciones escritas de origen de las importaciones léxicas, aun enfrentándose a su inseguridad, es lo que reconoce como propio de las lenguas donantes lo que le indica en qué dirección proceder.

15. Por el contrario, en español bonaerense se registra una correspondencia mimética entre $g$ ante vocal anterior (para importaciones del inglés, del francés y del italiano) y la consonante fricativa prepalatal sorda (Hipperdinger, 2015).

16. Son ejemplos las realizaciones orales recurrentemente registradas [mjúsli] para Müsli, y (entre los nombres propios) [mjúler] para Müller y [mjúnič] para Münich ( München - Múnich).

17. Dicha innovación posicional puede no restringirse a la de la representación grafémica de la pieza léxica de que se trate sino contrariar pautas generales de ubicación en la lengua donante, como lo ejemplifica $\operatorname{chipp}(s)$. 


\section{Síntesis y conclusiones}

Nos ocupamos en este artículo de la exageración de la extranjeridad en la configuración grafémica de importaciones léxicas sobre la base de materiales correspondientes a la región dialectal del español bonaerense, pertenecientes al área léxica de la gastronomía.

Encontramos que esa exageración, que resulta del intento de reproducir las configuraciones escritas de origen de voces procedentes de lenguas prestigiosas sobre las que se tiene un conocimiento limitado, adopta generalmente la forma de una amplificación grafémica, y más específicamente, de una duplicación consonántica. Esa amplificación, sin embargo, se opere o no en consonancia con alguna correspondencia mimética puntual convencionalizada, no habilita una lectura de los alternantes hipercorrectos que los separe de las realizaciones orales más extendidas en la variedad lingüística receptora, y entendemos que es por ello mismo por lo que se privilegia esa estrategia de (auto) corrección por sobre otras posibles.

Además, los recursos relevados en los alternantes hipercorrectos forman efectivamente parte de la escritura estandarizada de las lenguas donantes respectivas, lo que puede interpretarse en términos de limitación: cuando el impulso de preservar las configuraciones grafémicas de origen se enfrenta con un conocimiento insuficiente de dichas configuraciones, son los recursos grafémicos percibidos como característicos de las distintas lenguas donantes los que le indican al productor particular qué puede -y qué no- (hiper)corregir. Así, esos recursos son los medios de que se vale el productor para asegurarse de mantener no adaptadas las configuraciones escritas de las importaciones léxicas, un fin que revela el especialmente positivo valor simbólico asignado a la extranjeridad de las representaciones grafémicas de tales importaciones, al menos cuando provienen de lenguas donantes prestigiosas.

\section{Referencias}

Álvarez González, A. (2006). La variación lingüistica y el léxico: Conceptos fundamentales y problemas metodológicos. Hermosillo: Editorial de la Universidad de Sonora.

Bajo Pérez, E. (2008). El nombre propio en español. Madrid: Arco Libros.

Cabré Monné, T. (2010). La adaptación de préstamos en catalán. Ciclo de Conferencias 2010 "El espacio ibérico de las lenguas". Madrid: Instituto Cervantes. Recuperado de http:// www.cervantes.es/imagenes/File/lengua/jornadas/Teresa_Cabr_Monn_2010IC.pdf

Castillo Carballo, M. A. (2011). Voces extranjeras: de la realidad lingüística peninsular a la americana. Itinerarios, 13, 63-84. Recuperado de http://itinerarios.uw.edu.pl/ wp-content/uploads/2014/11/05-carballo.pdf

Corbella Díaz, D., y Real Cairós, A. M. (1997). La voz francesa entre la norma y el uso. Thélème. Revista Complutense de Estudios Franceses, 11, 229-237. Recuperado de http://revistas.ucm.es/index.php/THEL/article/view/THEL9797120229A/33953 
De Baere, J. (2012). Los extranjerismos en el español contemporáneo (Trabajo fin de Maestría). Universidad de Gent. Recuperado de http://lib.ugent.be/fulltxt/ RUG01/001/891/532/RUG01001891532_2012_0001_AC.pdf

Fontanella de Weinberg, M.B. (1987). El español bonaerense. Cuatro siglos de evolución lingüistica (1580-1980). Buenos Aires: Hachette.

Fontanella de Weinberg, M.B. (1994). "Una fugaza con fetas de panceta y provolone”: La incorporación léxica en español bonaerense. Estudios sobre el Español de la Argentina, III, 51-77.

Fontanella de Weinberg, M.B., Blanco de Margo, M., Hipperdinger, Y., Rigatuso, E., Suardíaz de Antollini, S., y Virkel de Sandler, A. (1991). Lengua e inmigración. Mantenimiento y cambio de lenguas inmigratorias. Bahía Blanca: Universidad Nacional del Sur.

Fontanella de Weinberg, M.B. (Ed.). (2000). El español de la Argentina y sus variedades regionales. Buenos Aires: Edicial.

Giménez Folqués, D. (2011). Normativa académica, adaptación y uso de los extranjerismos en el español actual (Tesis doctoral). Universidad de Valencia. Recuperado de http:// www.tesisenred.net/bitstream/handle/10803/78806/gimenez.pdf?sequence $=1$

Gómez Capuz, J. (1997). Towards a typological classification of linguistic borrowing (Illustrated with anglicisms in Romance languages). Revista Alicantina de Estudios Ingleses, 10, 81-94. Recuperado dehttps://rua.ua.es/dspace/bitstream/10045/5997/1/ RAEI_10_08.pdf

Gómez Capuz, J. (1998). El préstamo lingüistico. Conceptos, problemas y métodos. Valencia: Universitat de València.

Gómez Capuz, J. (2001). Estrategias de integración fónica de los anglicismos en un corpus de español hablado: asimilación, compromiso y efectos estructurales. Estudios de Lingüistica, 15, 1-85. Recuperado de http://rua.ua.es/dspace/ bitstream/10045/6222/1/EL_15_03.pdf

Gómez Molina, J.R. (2000). Transferencia y cambio de código en una comunidad bilingüe. Área metropolitana de Valencia (I y II). Contextos, XVII-XVIII, 309-360. Recuperado de https://dialnet.unirioja.es/servlet/articulo?codigo=853196

Haspelmath, M. (2009). Lexical borrowing: Concepts and issues. En M. Haspelmath y U. Tadmor (Eds.), Loanwords in the world's languages. A comparative handbook (pp. 35-54). Berlín: Walter de Gruyter.

Haugen, E. (1950). The analysis of linguistic borrowing. Language, 26, 210-231.

Hipperdinger, Y. (2015). Imitación simplificada (I). Correspondencias grafémicofonológicas miméticas en la realización oral de importaciones léxicas. Ponencia presentada en el II Congreso de la Delegación Argentina de la ALFAL y en las VII Jornadas Internacionales de Investigación en Filología Hispánica, La Plata.

Iturrioz, P. (2006). Lenguas propias-lenguas ajenas. Buenos Aires: Libros del Zorzal. 
Lasry, L. (2014). ¿Cómo pronunciamos los apellidos? La realización oral de apellidos de origen italiano en noticieros televisivos de la ciudad de Bahía Blanca. Ponencia presentada en las IV Jornadas de Lengua, Literatura y Comunicación. Viedma.

Leal Cabrera, L.A. (2001). Acomodamiento fonético de los anglicismos en el español de Cuba. Ponencia presentada en el 7th International Symposium on Social Communication, Santiago de Cuba.

Mateescu, M. (2013). Ortografía de los anglicismos. Grados y clases de adaptación. Analele Facultatii de Limbi si Literaturi Straine, 1, 1-7. Recuperdo de http://aflls. ucdc.ro/en/I_2013/4ORT.pdf

Myers-Scotton, C. (2006). Multiple voices. An introduction to bilingualism. Oxford: Blackwell.

Pratt, C. (1980). El anglicismo en el español peninsular contemporáneo. Madrid: Gredos.

Reyes Padilla, V. (2009). Anglicismos difundidos por la prensa y la publicidad (Trabajo fin de Licenciatura). Universidad Nacional Mayor de San Marcos. Recuperado de http://cybertesis.unmsm.edu.pe/bitstream/cybertesis/956/1/Reyes_pv.pdf.

Rodríguez González, F. (2008). Variaciones grafemáticas de los anglicismos en los medios de comunicación: tendencias y factores condicionantes. En T. Gibert Maceda y L. Alba Juez (Eds.), Estudios de Filología Inglesa. Homenaje a la Dra. Asunción Alba Pelayo (pp. 269-283). Madrid: Universidad Nacional de Educación a Distancia.

Romaine, S. (1995). Bilingualism (2a ed.) Oxford: Blackwell.

Weinreich, U. ([1953] 1968). Languages in contact. Findings and problems (9 $\left.9^{\mathrm{a}} \mathrm{ed}.\right)$. The Hague: Mouton.

Winford, D. (2003). An introduction to Contact Linguistics. Oxford: Blackwell.

Winter-Froemel, E. (2008). Studying loanwords and loanword integration: Two criteria of conformity. Newcastle Working Papers in Linguistics, 14, 156-176. Recuperado de http://www.ncl.ac.uk/linguistics/assets/documents/11.Esme_Winter-Froemel-FT.pdf 